Preprint LBNL-39661

\title{
Probing the quantum-mechanical equivalent-photon spectrum for electromagnetic dissociation of relativistic uranium projectiles
}

\author{
Th. Rubehn * \\ Nuclear Science Division, Ernest Orlando Lawrence Berkeley National Laboratory, \\ University of California, Berkeley, California 94720 \\ W. F. J. Müller, W. Trautmann \\ Gesellschaft für Schwerionenforschung (GSI), Planckstraße 1, D-64291 Darmstadt, Germany
}

(November 10, 2018)

\begin{abstract}
Electromagnetic fission cross sections for the reactions $U$ $+(\mathrm{Be}, \mathrm{C}, \mathrm{Al}, \mathrm{Cu}, \mathrm{In}, \mathrm{Au}, \mathrm{U})$ at $E / A=0.6$ and $1.0 \mathrm{GeV}$ are compared to theoretical calculations using recently proposed quantum-mechanical equivalent-photon spectra. In contrast to semi-classical calculations, systematically lower cross sections are obtained that cannot reproduce the experimental results. Furthermore, we point out that the study of electromagnetic fission cross sections or electromagnetic 1-neutron removal cross sections alone cannot provide unambiguous information on the excitation of the double giant dipole resonance.
\end{abstract}

PACS number(s): 25.75.-q, 25.85.Ge, 27.90.+b

The availability of relativistic heavy-ion beams has enabled systematic studies of electromagnetic excitation processes in nuclei [1]. Generally, the electromagnetic interaction between the projectile and the target nuclei is described by the exchange of virtual photons. Due to the almost quadratic dependence on the charge of the reaction partner and due to the fast time variation of the Lorentz-contracted electromagnetic field, electromagnetic cross sections are rather large for relativistic heavyion collisions; projectile energies of $\sim 1 \mathrm{GeV} /$ nucleon allow for an effective excitation of the giant resonances $(10-30 \mathrm{MeV})$. When the nucleus is excited above its particle emission threshold, or, in the case of fission above its fission threshold, it may then dissociate according to the appropriate branching ratio. Experimentally, measurements have investigated various decay branches, like $\gamma$-rays, neutron emission, and fission, see, e.g., Refs. [1 20]. Almost exclusively, all the experimental data have been compared with calculations using the semi-classical Weizsäcker-Williams method of virtual photons 21 24] which has been shown to give an appropriate description of the process [24].

Recently, Benesh, Hayes, and Friar have presented new quantum-mechanical descriptions of the equivalentphoton spectra for electromagnetic heavy-ion collisions

*Electronic address: TRubehn@lbl.gov
25. This recent work has extended previous studies 26, 27 by examining the sensitivity of nuclear structure inputs. Electromagnetic excitation cross sections are calculated using the first Born approximation. Finally, a model is presented that gives simple quantum-mechanical expressions for the E1 and E2 "equivalent-photon spectrum" which can be used with measured photoabsorption cross sections in exactly the same way as the usual semi-classical expression. Electromagnetic dissociation (EMD) cross sections for a specific decay channel $\Psi$ can then - generally - be expressed by:

$$
\sigma_{\mathrm{EMD}}^{\Psi}=\int\left(\sigma_{\gamma, \Psi}^{\mathrm{E} 1} n^{\mathrm{E} 1}(\omega)+\sigma_{\gamma, \Psi}^{\mathrm{E} 2} n^{\mathrm{E} 2}(\omega)\right) d \omega,
$$

where $\omega$ is the photon energy, $\sigma_{\gamma}$ is the photodissociation cross section and $n(\omega)$ is the photon spectrum generated by the collision partner. The indices E1 and E2 indicate the multipolarities. While most semi-classical calculations make use of a cutoff parameter, $b_{\min }$, in coordinate space to account for electromagnetic contributions only, the quantum-mechanical description introduces a cutoff parameter in momentum space [25]:

$$
\begin{aligned}
& q_{\max }=1 / b_{\min }= \\
& \quad\left[1.34\left(A_{\mathrm{P}}^{1 / 3}+A_{\mathrm{T}}^{1 / 3}-0.75\left(A_{\mathrm{P}}^{-1 / 3}+A_{\mathrm{T}}^{-1 / 3}\right)\right)\right]^{-1}
\end{aligned}
$$

where $A_{\mathrm{P}}$ and $A_{\mathrm{T}}$ are the mass numbers of the projectile and target, respectively [28]. It has been shown that the used parameterization of $b_{\min }$ allows for a good description of the total nuclear reaction cross section [29].

In this paper, we will not discuss the quantummechanical ansatz per se which has been presented in Ref. [25]; this issue will be addressed elsewhere [30]. However, we shall apply the given quantum-mechanical virtual photon spectra to calculate electromagnetic dissociation cross sections of relativistic heavy-ion collisions. Comparisons between these calculations and semiclassical calculations on one hand and experimental results on the other hand will be discussed. In particular, it will be shown that due to uncertainties in various input parameters, no unambiguous conclusions on the excitation of the double giant dipole resonance (DGDR) can be drawn by only investigating the electromagnetic $1 \mathrm{n}$ removal cross sections as it has been done in Ref. 25]. 


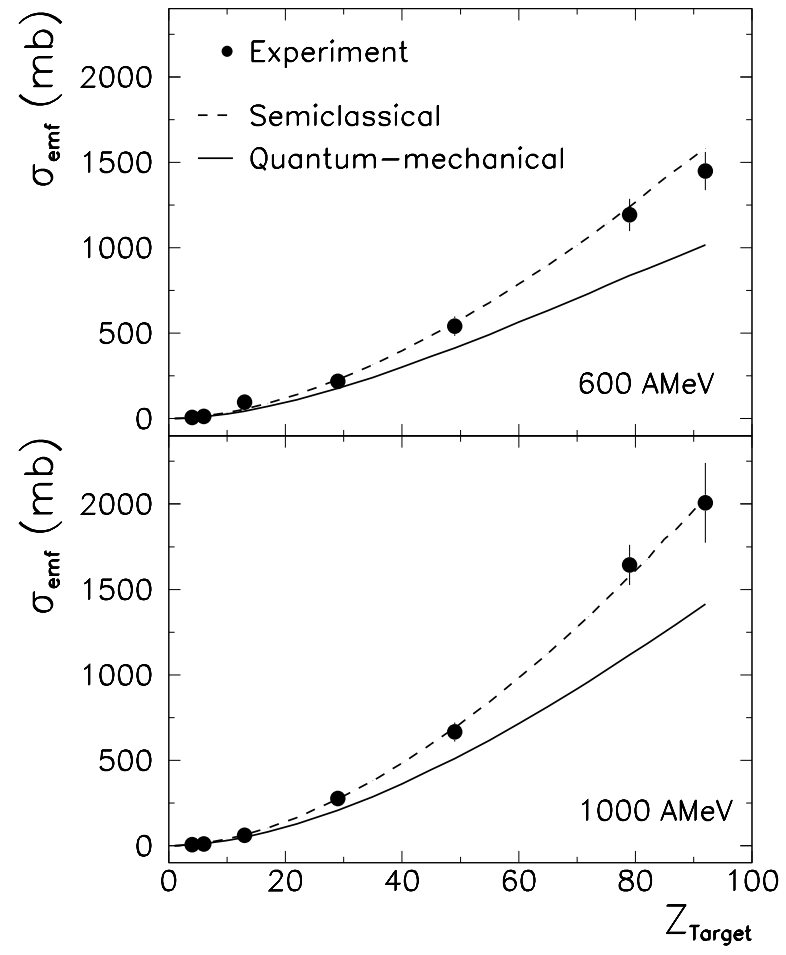

FIG. 1. Electromagnetic fission cross sections for the reactions ${ }^{238} \mathrm{U}+(\mathrm{Be}, \mathrm{C}, \mathrm{Al}, \mathrm{Cu}, \mathrm{In}, \mathrm{Au}, \mathrm{U})$ at $\mathrm{E} / \mathrm{A}=600$ and $1000 \mathrm{MeV}$ (from Ref. 17]). For comparison, theoretical results are shown using both the semiclassical (dotted line) [17] and the quantum mechanical descriptions (full line) 25] of the photon spectra.

We will first focus on the comparison of EMD cross sections. In a previous work, electromagnetic fission (EMF) of ${ }^{238} \mathrm{U}$ projectiles has been experimentally studied 17] using the ALADIN spectrometer at the heavy-ion synchrotron at GSI, Darmstadt. Seven different targets (Be, $\mathrm{C}, \mathrm{Al}, \mathrm{Cu}, \mathrm{In}, \mathrm{Au}$, and $\mathrm{U}$ ) have been bombarded at 0.6 and $1.0 \mathrm{GeV} /$ nucleon. Experimental details on the measurements and the setup can be found in Refs. 17, 31, 32. In Fig. 1, the deduced electromagnetic fission cross sections taken from Ref. [17] are shown as a function of the charge number of the target for both bombarding energies.

For comparison, both the semi-classical and the quantum-mechanical calculations have been performed in the same fashion using Eq. 1. The use of parameterizations of the photodissociation cross sections $\sigma_{\gamma \text {,total }}$ [33], the fission probability and the cutoff parameter where nuclear interactions become dominant [28] has already been discussed previously [17].

While the semi-classical calculations can well reproduce the experimental data, the quantum-mechanical calculations give significantly lower cross sections. This is due to a reduced photon flux in the quantum-mechanical description. Note that this discrepancy is larger than the uncertainties connected with the choice of the cut- off parameter or the resonance parameters (see, e.g., Refs. [17, 18]): the Kox parametrization [34 of the cutoff parameter results in $\sim 15 \%$ lower cross sections, even the use of photodissociation cross sections [35] which are known to be systematically high compared to other measurements [36 39], results in only $15 \%$ higher cross sections. The experimental data can only be reproduced by the quantum-mechanical calculations when the cutoff parameter in momentum space $q_{\max }$ is artificially increased by $30 \%$. For the reaction ${ }^{238} \mathrm{U}+{ }^{238} \mathrm{U}$, this results in a value of $b_{\min }=12.5 \mathrm{fm}$ compared to $b_{\min }=16.3 \mathrm{fm}$ from Eq. 2. This significantly lower value seems inappropriate in terms of the total absorption radius and is not in agreement with previous works investigating total reaction cross sections (see, e.g., Ref. [29]). While the equivalence between the sharp and smooth cutoff $b_{\min }$ has been shown to be valid in coordinate space [11], it seems to be invalid for the cutoff $q_{\max }$ in momentum space [30].

Furthermore, calculations predict nearly the same cross sections for electromagnetic fission, whether or not the possibility of two phonon excitation is included. This is due to the fact that the higher fission probability in the energy regime of the DGDR is to a large extent compensated by the redistribution of cross section from 1phonon to that of 2-phonon excitation 117. Therefore, the results of the calculations are almost independent of the strength of the DGDR as long as EMF cross sections are discussed. The comparison of calculated EMF cross sections with experimental data is thus virtually independent of the excitation of the DGDR. We conclude that the quantum-mechanical photon spectra presented in Ref. 25] are - in contrast to semi-classical photon spectra - not able to describe the experimental results.

Electromagnetic fission cross sections alone do not allow one to draw conclusions on the excitation of the DGDR. This is also true for the 1-neutron removal cross sections measured in Refs. 迆 8,10 12. It has been shown previously that the $1 \mathrm{n}$ cross sections calculated using multiphonon excitations of the GDR differ only insignificantly from simple calculations based upon the excitation of the 1-phonon state only [10].

Many efforts have been made to measure quantities which permit a more direct test of the excitation of the double GDR [2, $4,10,12,17,20]$. In the following, we briefly review some of these observables:

1) The asymmetry of the fission fragment charge distribution is known to be very sensitive to the excitation energy distribution. The asymmetry is usually expressed by the peak-to-valley ratio of the double humped charge distribution. In two independent experiments, a peakto-valley ratio of $7.6 \pm 2.6$ and $7.1 \pm 1.0$, respectively, has been found [17,19]. Calculations show that the excitation of the single phonon states alone would result in a significantly higher peak-to-valley ratio of $16 \pm 3$, while calculations which account for the excitation of the DGDR can reproduce the experimental findings. Therefore, the low peak-to-valley ratio has been interpreted as a clear evidence of the DGDR excitation. This conclusion is also 
supported by the measurement of the proton odd-even effect of the fission fragment distribution [17. We note that these quantities are completely independent of the integrated EMF cross sections.

2) Aumann et al. have studied electromagnetic dissociation by measuring $1 \mathrm{n}-5 \mathrm{n}$ neutron removal cross sections for various reactions 110,12 . Studying the $2 \mathrm{n}, 3 \mathrm{n}, 4 \mathrm{n}$, and $5 \mathrm{n}$ removal cross sections allows for a sensitive test of the contributions from higher excitation energies. It has been shown that these data cannot be understood without the excitation of the DGDR which accounts for the largest fraction of the cross section in the competing channels 10,12].

3) Recently, Boretzky et al. have reported conclusive evidence for the excitation of the DGDR in ${ }^{208} \mathrm{~Pb}$ [20]. By measuring both $\gamma$-rays and neutrons, the excitation energy was reconstructed and differential cross sections $\mathrm{d} \sigma / \mathrm{d} E^{*}$ were deduced for several targets. These results allow for the comparison of the differential cross sections as a function of excitation energy with calculations and provide a direct test of the strength of the DGDR.

The experimental evidence listed above contradicts the theoretically deduced statement by Benesh, Hayes, and Friar 25] saying that the quantum-mechanical photon spectra leave little room for multiphonon mechanisms.

In conclusion, we have applied the quantummechanical equivalent photon spectra presented in Ref. 25] in order to make comparisons with experimental results. It turns out that electromagnetic fission cross sections of uranium in the energy regime of $\sim 1 \mathrm{GeV} /$ nucleon are independent of the strength of the DGDR and thus permit tests of theoretical calculations without complications that may result from multiphonon excitations. Comparisons with experimental electromagnetic fission data show that, due to the significantly lower photon flux of the quantum-mechanical description, the experimental EMF cross sections cannot be described by the calculations. Since uncertainties in the parametrizations are significantly smaller, we conclude that the quantum-mechanical photon spectrum presented in Ref. 25] is not able to describe the experimental results. Furthermore, no conclusive information on the excitation of multiphonon mechanisms can be drawn from the study of cross sections of electromagnetic fission or $1 \mathrm{n}$ removal only.

This work was supported by the Director, Office of Energy Research, Office of High Energy and Nuclear Physics, Nuclear Physics Division of the US Department of Energy, under contract DE-AC03-76SF00098.

[1] H. Emling, Prog. Part. Nucl. Phys. 33, 729 (1994) and references therein.
[2] J. Ritman et al., Phys. Rev. Lett. 70, 533 (1993).

[3] R. Schmidt et al., Phys. Rev. Lett. 70, 1767 (1993).

[4] E. Wajda et al., Nucl. Phys. A 569, 141c (1994).

[5] M. T. Mercier, J. C. Hill, F. K. Wohn, C. M. McCullough, M. E. Nieland, J. A. Winger, C. B. Howard, S. Renwick, D. K. Matheis, and A. R. Smith, Phys. Rev. C 33, 1655 (1986).

[6] J. C. Hill, F. K. Wohn, J. A. Winger, M. Khayat, K. Leininger, and A. R. Smith, Phys. Rev. C 38, 1722 (1988).

[7] J. C. Hill, F. K. Wohn, J. A. Winger, M. Khayat, M. T. Mercier, and A. R. Smith, Phys. Rev. C 39, 524 (1989).

[8] J. C. Hill and F. K. Wohn, Phys. Rev. C 39, 2474 (1989).

[9] J. C. Hill, F. K. Wohn, D. D. Schwellenbach, and A. R. Smith, Phys. Lett. B 273, 371 (1991).

[10] T. Aumann, J. V. Kratz, E. Stiel, K. Sümmerer, W. Brüchle, M. Schädel, G. Wirth, and J. C. Hill, Phys. Rev. C 47, 1728 (1993).

[11] T. Aumann, C. A. Bertulani, and K. Sümmerer, Phys. Rev. C 51, 416 (1995).

[12] T. Aumann et al., Z. Phys. A 352, 163 (1995).

[13] P.L. Jain, M.M. Aggarwal, M.S. El-Nagdy, and A.Z.M. Ismail, Phys. Rev. Lett. 52, 1763 (1984).

[14] D.E. Greiner, H. Crawford, P. J. Lindstrom, J. M. Kidd, D. L. Olson, W. Schimmerling, and T. J. M. Symons, Phys. Rev. C 31, 416 (1985).

[15] M.L. Justice, Y. Blumenfeld, N. Colonna, D. N. Delis, G. Guarino, K. Hanold, J. C. Meng, G. F. Peaslee, G. J. Wozniak, and L. G. Moretto, Phys. Rev. C 49, R5 (1994).

[16] S. Polikanov et al., Z. Phys. A 350, 221 (1994).

[17] Th. Rubehn et al., Z. Phys. A 353, 197 (1995).

[18] Th. Rubehn et al., Phys. Rev. C 53, 3143 (1996).

[19] P. Armbruster et al., Z. Phys. A. 355, 191 (1996).

[20] K. Boretzky et al., Phys. Lett. B 384, 30 (1996).

[21] E. Fermi, Z. Phys. 29, 315 (1924).

[22] C. F. von Weizsäcker, Z. Phys. 88, 612 (1934).

[23] E. J. Williams, Phys. Rev. 45, 729 (1934).

[24] C. A. Bertulani and G. Baur, Phys. Rep. 163, 299 (1988).

[25] C. J. Benesh, A. C. Hayes, and J. L. Friar, Phys. Rev. C 54, 1404 (1996).

[26] C. J. Benesh and J. L. Friar, Phys. Rev. C 48, 1285 (1993).

[27] C. J. Benesh and J. L. Friar, Phys. Rev. C 50, 3167 (1994).

[28] C.J. Benesh, B.C. Cook, and J.P. Vary, Phys. Rev. C 40, 1198 (1989).

[29] M. Hesse et al., Z. Phys. A. 355, 69 (1996).

[30] G. Baur and C. A. Bertulani, Phys. Rev. C (submitted), (Gesellschaft für Schwerionenforschung, GSI-96-54, 1996 and xxx.lanl.gov, e-print nucl-th/9611025, 1996).

[31] Th. Rubehn et al., Phys. Rev. C 53, 993 (1996).

[32] A. Schüttauf et al., Nucl. Phys. A607, 457 (1996).

[33] A. Veysièrre, H. Beil, R. Bergère, P. Carlos, A. Lepetre, and K. Kernbath, Nucl. Phys. A199, 45 (1973).

[34] S. Kox et al., Phys. Rev. C35, 1678 (1987).

[35] J. T. Caldwell, E. J. Dowdy, B. L. Berman, R. A. Alvarez, and P. Meyer, Phys. Rev. C21, 1215 (1980).

[36] W. Wilke, U. Kneissl, Th. Weber, H. Ströher, L. S. Cardman, P. T. Debevec, S. D. Hobbit, R. T. Jones, and A. 
M. Nathan, Phys. Rev. C42, 2148 (1990).

[37] H. Ries, G. Mank, J. Drexler, R. Heil, K. Huber, U. Kneissl, R. Ratzek, H. Ströher, T. Weber, and W. Wilke, Phys. Rev. C29, 2346 (1984).

[38] Th. Weber, R. D. Heil, U. Kneissl, W. Wilke, Th. Kihm, K. T. Knöpfle, and H. J. Emrich, Nucl. Phys. A510, 1 (1990).

[39] G. M. Gurewich, L. E. Lazareva, V. M. Mazur, G. V. Solodukhov, and B. A. Tulupov, Nucl. Phys. A273, 326 (1976). 